\title{
Aphid Viruses: A Brief View of a Long History
}

\author{
Ya Guo, Ning Ji, Lisha Bai, Juntian Ma and Zhaofei Li * \\ State Key Laboratory of Crop Stress Biology for Arid Areas, Key Laboratory of Northwest Loess Plateau Crop Pest \\ Management of Ministry of Agriculture, College of Plant Protection, Northwest A\&F University, Yangling, China
}

OPEN ACCESS

Edited by:

Chunsheng Hou,

Institute of Bast Fiber Crops

(CAAS), China

Reviewed by:

Xiaobin Shi,

Hunan Academy of Agricultural

Sciences, China

Hongxia Zhao,

Guangdong Institute of Applied Biological Resources, China

*Correspondence:

Zhaofei Li

zhaofeili@nwsuaf.edu.cn

Specialty section:

This article was submitted to Insect Health and Pathology,

a section of the journal

Frontiers in Insect Science

Received: 31 December 2021

Accepted: 31 January 2022

Published: 24 February 2022

Citation:

Guo Y, Ji N, Bai L, Ma J and Li Z (2022) Aphid Viruses: A Brief View of a

Long History.

Front. Insect Sci. 2:846716.

doi: 10.3389/finsc.2022.846716
Aphids are common agricultural pests with a wide range of hosts from agriculture to forestry plants. As known, aphids also serve as the major vectors to transmit plant viruses. Although numerous studies have focused on interactions between aphids and plant viruses, little is known about the aphid viruses, i.e., the insect viruses that are infectious to aphids. In the past four decades, several aphid viruses have been identified in diverse aphid species. In this review, we present a brief view of the aphid pathogenic viruses from several aspects, including classification of aphid viruses and characters of the viral genome, integration of viral sequences in host genomes, infection symptoms and influence on aphids, as well as host range and transmission modes. Taken together, these studies have increased our understanding of the rarely known aphid viruses, and will potentially contribute to the development of new strategies for controlling aphid populations.

\section{Keywords: aphids, densovirus, bunyavirus, dicistrovirus, flavivirus, iflavirus, mesonivirus}

\section{INTRODUCTION}

Aphids belong to Hemiptera, Aphidoidea. Currently, over 5,000 species of aphids in about 500 genera have been recognized (1). The individuals of this large group of insects are widely distributed in subtropical and temperate regions of the Northern hemisphere. They have a high reproductive potential and phenotypic plasticity. Within their complex life cycles, most species adopt a seasonal periodic pathenogenesis with alternating asexual and sexual stages and generate winged (alatae) and unwinged (apterae) morphs. As polyphagous phloem-feeders, aphids are the major insect pests on a diversity of crops, such as cereals, flowers, fruits, and vegetables (2-5). During ingesting phloem sap from host plants, aphids can probe almost all plant tissues with their stylets and acquire or inoculate the virus within plants. As the most efficient vectors of plant viruses, aphids are estimated to transmit nearly over $30 \%$ of insect-borne viruses $(6,7)$. Additionally, aphids honeydew induce sooty molds grow on leaves and hinder the photosynthetic activities of plants (4).

For a long time, numerous studies have demonstrated the relationship of aphids and plant viruses, with emphasis on interactions between plant viruses, aphids, and plants, as well as the molecular mechanisms underlying these interactions $(5,6,8)$. In contrast, insect viruses that are infectious to aphids receive little attention. In the 1960s, virus-like particles have been described in Myzus persicae and Rhopalosiphum maidis $(9,10)$. However, the first aphid virus, Rhopalosiphum padi virus (RhPV), has been characterized by D'Arcy et al. (11). Currently, several aphid pathogenic viruses have been identified in diverse aphid species. Based on nucleotide sequences, these viruses can be divided into two categories: (1) DNA viruses: these viruses include Dysaphis plantageinea densovirus (DplDV), Myzus persicae densovirus (MpDV), and Myzus persicae nicotianae densovirus (MpnDV), which are small DNA viruses and grouped into Densovinae in 
the family Parvoviridae; (2) RNA viruses: these viruses are further classified into different groups: (i) Bunyaviruses: including Aphid bunyavirus 1 (ABV-1) and Aphis citricidus bunyavirus (AcBV); (ii) Dicistroviruses: including Aphid lethal paralysis virus (ALPV) and RhPV; (iii) Flaviviruses: including Macrosiphum euphorbiae virus 1 (MeV-1); (iv) Iflaviviruses: including Brevicoryne brassicae virus (BrBV); (v) Mesoniviruses: including Aphis citricidus meson-like virus (AcMSV); (vi) Unclassified RNA viruses: including Aphis glycines virus 1 (ApGlV1), Aphis glycines virus 2 (ApGlV2), Acyrthosiphon pisum virus (APV), and Rosy apple aphid virus (RAAV) (Table 1). In this article, we present a brief view of the aphid viruses from several aspects, which include the taxonomy of the virus, characters of the viral genome, integration of certain virus-derived sequences in aphid genomes, infection symptoms and mechanisms, as well as host range and transmission modes.

\section{DNA VIRUSES}

\section{Densoviruses}

\section{Classification and Characters of the Genome of Aphid Densoviruses}

Densoviruses are non-enveloped small DNA viruses. The virus particle is icosahedral symmetry with the diameter of about $25 \mathrm{~nm}$. The genome of densoviruses is single-stranded linear DNA with the length of $4-6 \mathrm{~kb}$. Termini of both ends of the genome are locked with a short duplex haripin telomeres ( $200 \mathrm{bp})$, which involved in DNA replication. Densoviruses contains two sets of genes encoding 2-3 nonstructural (NS) and 2-4 capsid (VP) proteins (25). Currently, densoviruses are grouped into Densovirinae in the family Parvoviridae. The Densovirinae subfamily is further divided into five genera: Ambidensovirus, Brevidensovirus, Hepandensovirus, Iteradensovirus, and Penstyldensovirus. Ambidensoviruses have coding capacities in both directions of the strand. The NS genes are located at the 5'-proximal of the sense strand, while the VP genes are derived from the 5'-proximal of the antisense strand. In comparison, other densoviruses are monosense viruses, which encode NS proteins at the 5 '-terminus, and VP proteins at the 3 '-terminus of the same strand (26).

In 2003, van Munster et al. first identified $\mathrm{MpDV}$ in $M$. persicae (13). Like other members of Densovirinae, MpDV particles are isocochatral and have a diameter of $\sim 20 \mathrm{~nm}$. MpDV genome lack of the inverted terminal repeat (ITR) is 5,499 nt and predicted to encode five ORFs. Three predicted ORFs are located at the $5^{\prime}$-proximal region of the sense strand and encode three NS proteins (NS1-NS3) with different sizes (NS1, 698aa; NS2, 225aa; and NS3, 97aa), whereas two ORFs are located at the $5^{\prime}$-proximal region of the antisense strand and predicted to encode five capsid protein (VP) with the size of $57-92 \mathrm{kDa}$ $(13,27)$ (Figure 1A). In 2009, Ryabov et al. identified DplDV in the rosy apple aphid Dysaphis plantageinea (12). The genome of DplDV is 4,979 nt and predicted to encode four ORFs. Two ORFs located on the 5'-proximal of the sense strand encode NS1 (710aa, encoded by ORF1) and NS2 (414 aa, encoded by ORF2), whereas the other two ORFs located on the antisense strand encode VP proteins (12) (Figure 1A). Similarly, a recently identified MpnDV genome possesses three putative ORFs with two encoding NS1 and NS2 proteins on the sense strand, as well as the third one encoding a VP protein on the antisense strand (Figure 1A). Genome sequence alignment analysis showed that $\mathrm{MpnDV}$ was closely related with $\mathrm{MpDV}$, and both viruses may represent different strains of a same virus species (14). Based on the phylogenetic analysis and transcription assay, these aphid densoviruses are grouped in the genus Ambidensovirus $(13,14)$ (Table 1).

Additionally, partial sequence $(2,668 \mathrm{nt})$ of a new densovirus Macrosiphum euphorbiae virus $2(\mathrm{MeV}-2)$ was retrieved from the transcriptome data of the potato aphid Macrosiphum euphorbiae. Although sequence alignment indicated that $\mathrm{MeV}-2$ was closely related with DplDV, the detailed molecular characters and classification of this virus are not clear (28).

\section{Integration and Transcription of Densovirus-Derived Sequences in Aphid Genomes}

Retroviral and non-retroviral sequences are commonly integrated into insects, plants, and vertebrates genomes by different replication strategies (29-33). In long-term arm races between viruses and hosts, these endogenous viral elements (EVEs) either serve as the fossil record of past virus infection or play functional roles in diverse aspects in regulating host genome stability, immune response, and environmental fitness (29-31).

Aphids have remarkable wing plasticity. In response to high population densities or poor plant quality, the wing dimorphism allows asexual aphids to produce winged offspring that essentially promote aphid dispersal $(2,34)$. Prior studies indicated that EVEs derived from densoviruses integrated into the genome of A. pisum (35). Recently, the transcription of two EVEs (Apns1, Apns-2), which showed high similarities to the NS protein sequences of MpDV and DplDV, were found to be up-regulated in response to aphid crowding in those wing highly inducible genotypes of $A$. pisum. RNAi knockdown the transcription of those genes substantially reduce the percentage of winged morphs of the genotype, suggesting that these densovirus-derived EVEs play critical roles in regulating wing plastic traits of pea aphids (36). Recent findings indicated that 21 desovirus-derived EVEs were integrated into 10 scaffords of the $M$. persicae genome (37). Although the significance of these EVEs is not clear, the relative high transcription level of some EVEs and the moderate effect of MpDV on growth and development of $M$. persicae suggest that the integration of desovirus-derived EVEs may regulate fitness of the virus and aphids $(27,37)$.

\section{Infection Impact and Cytopathology of Aphid Densoviruses}

Prior studies showed that the aphids (M. persicae and $D$. plantaginea) infected with MpDV or DplDV had a dramatic reduction of their weight, fecundity, and development time $(12,27)$. However, MpDV infection has no significant effect on the intrinsic growth rate of the $M$. persicae population (27). Intriguingly, DplDV infection led the asexual clones of $D$. plantaginea to produce a high percentage of winged offspring (12). This phenomenon is consistent with that observed in 
TABLE 1 | Classification and characters of aphid viruses.

\begin{tabular}{|c|c|c|c|c|c|c|}
\hline \multirow[t]{2}{*}{ Virus category } & \multicolumn{3}{|c|}{ Virus classification } & \multirow[t]{2}{*}{ Env\& } & \multirow[t]{2}{*}{ Genome” (GenBank no.) } & \multirow[t]{2}{*}{ References } \\
\hline & Family/subfamily & Genus & Virus & & & \\
\hline \multirow[t]{3}{*}{ Densovirus } & Parvoviridae/Densovirinae & Ambidensovirus & Dysaphis plantageinea densovirus & - & (+)ssDNA, 4979 nt (EU851411) & (12) \\
\hline & & & Myzus persicae densovirus & - & (+)ssDNA, 5499 nt (AY148187) & $(13)$ \\
\hline & & & Myzus persicae nicotianae densovirus & - & (+)ssDNA, 5480 nt (KT239104) & $(14)$ \\
\hline \multirow[t]{2}{*}{ Bunyavirus } & Phasmaviridae & NA & Aphid bunyavirus 1 & + & $\begin{array}{l}\text { (-)ssRNA, L/M/S*: 7317/6626/1874 } \\
\text { nt (NA) }\end{array}$ & $(15)$ \\
\hline & Phenuiviridae & NA & Aphis citricidus bunyavirus & + & $\begin{array}{l}\text { (-)ssRNA, L/M/S*:7037/ 3462/1163 } \\
\text { nt (MN163034, MN163035, } \\
\text { MN163036) }\end{array}$ & $(16)$ \\
\hline \multirow[t]{2}{*}{ Dicistrovirus } & Dicistroviridae & Cripavirus & Aphid lethal paralysis virus & - & (+)ssRNA, 9812 nt (NC_004365) & $(17)$ \\
\hline & & & Rhopalosiphum padi virus & - & (+)ssRNA, 10011 nt (AF022937) & (18) \\
\hline Flavivirus & Flaviviridae & Flavivirus & Macrosiphum euphorbiae virus 1 & + & (+)ssRNA, 22780 nt (KT309079) & (19) \\
\hline Iflavirus & Iflaviridae & Iflavirus & Brevicoryne brassicae virus & - & (+)ssRNA, $10161 \mathrm{nt}($ EF517277) & (20) \\
\hline Mesonivirus & Mesoniviridae & NA & Aphis citricidus meson-like virus & & (+)ssRNA, 20300 nt (MN961271) & $(21)$ \\
\hline \multirow[t]{4}{*}{ Unclassified RNA virus } & NA & NA & Acyrthosiphon pisum Virus & - & (+)ssRNA, 10016 nt (AF024514) & (22) \\
\hline & & & Aphis glycines virus 1 & - & (+)ssRNA, 8680 nt (KM015260) & (23) \\
\hline & & & Aphis glycines virus 2 & - & (+)ssRNA, 4850 nt (KR912180) & $(24)$ \\
\hline & & & Rosy apple aphid virus & - & (+)ssRNA, 9992 nt (DQ286292) & $(12)$ \\
\hline
\end{tabular}

\&Env represents the envelope of virus.

" $L / M / S$ represents the large, medium, and small segments of the virus genome.

\#Genome: most of the virus genomes are incomplete.

NA, not available.

A. pisum that RNAi knockdown the expression of densovirusderived EVEs impact the production of winged morphs (36). These studies suggest that densovirus infection or integration of desovirus-derived EVEs regulates the wing plasticity of aphids.

Currently, little is known about infection mechanisms of aphid densoviruses. Earlier transmission electron microscopy (TEM) studies showed that MpDV could be only replicated in the nuclei of cells of the anterior part, i.e., the stomach of the midgut of $M$. persicae. The nuclei of infected midgut cells usually expanded twice in size compared with that of healthy cells, and vesicles containing progeny virions were observed clustering in the perinuclear space between the inner and outer nuclear membranes. In addition, large amount of progeny virions were found in large and spherical vesicles in the cytoplasm of infected cells (27).

\section{Host Range and Transmission Modes of Aphid Densoviruses}

The host range of aphid densoviruses is lack of systematic investigation. Earlier studies indicated that $\mathrm{MpDV}$ could infect only $M$. persicae and possibly the whitefly Trialeurodes vaporariorum (family Aleyrodidae), but not infect the other aphids, such as A. pisum, Metopolophium dirhodum, Rhopalosiphum padi, or Schizaphis graminum (27).

Few studies revealed that aphid densoviruses including DplDV, MpDV, and the putative MeV-2 can be transmitted vertically from infected adults to nymphs (12, 27, 28). Interestingly, although the majority of nymphs produced by infected aphids were tested positively, a proportion of nymphs were still virus free for DplDV and $\operatorname{MpDV}(12,27)$. Horizontal transmission of densoviruses from infected aphids to healthy ones was also observed in different aphids. In addition, prior studies indicated that host plants play important roles in horizontal transmission of aphid densoviruses. MpDV could be detected in healthy aphids that placed on leaves far from the infected-aphids infested leaves $(12,27,28)$. Although no evidence indicate that these viruses could replicate in plants, current data suggest that aphid densoviruses may circulate in plants. The plant may serve as reservoirs for virus transmission or they may utilize the virus as a weapon defense against aphids (38).

\section{RNA VIRUSES}

\section{Bunyaviruses}

\section{Classification and Characters of the Genome of Aphid Bunyaviruses}

Bunyaviruses are a group of enveloped viruses that have spherical virions with the diameter of $80-120 \mathrm{~nm}$. Most bunyavirus genomes consist of three segments of single-stranded, negativesense RNA: large (L), medium (M), and small (S), which separately encodes an RdRp, envelope glycoprotein(s), and viral capsid proteins (39). Bunyaviruses can infect various hosts including invertebrates, plants, and vertebrates, and mostly are transmitted by invertebrate vectors. Since this group contains highly abundant viruses and new species are continuously found, the taxonomy of bunyavirus has been re-defined by the International Committee on Taxonomy of Viruses (ICTV) from the family Bunyaviridae to the order Bunyavirales in 2017 (40). 
A
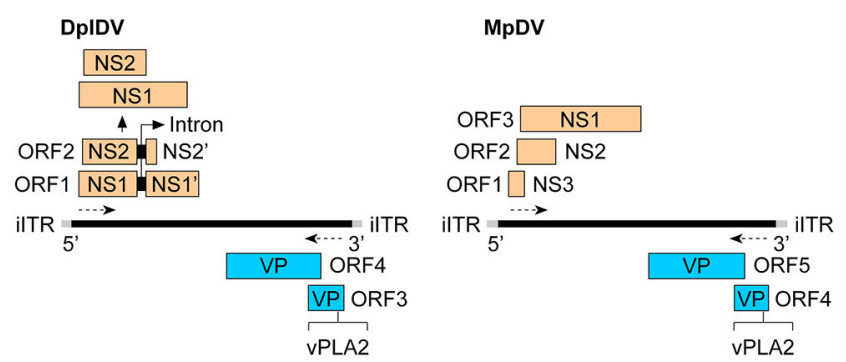

MpnDV

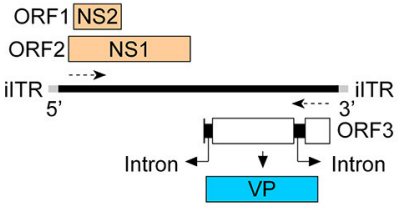

B

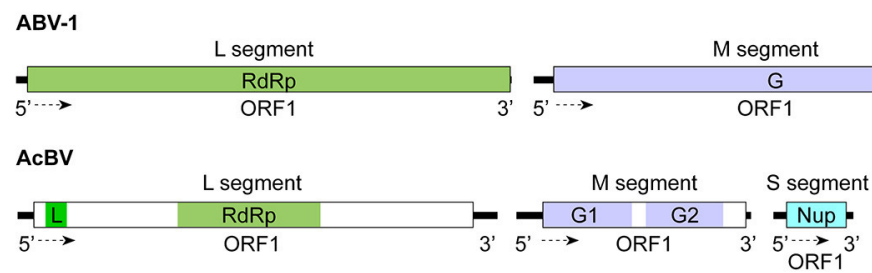

C

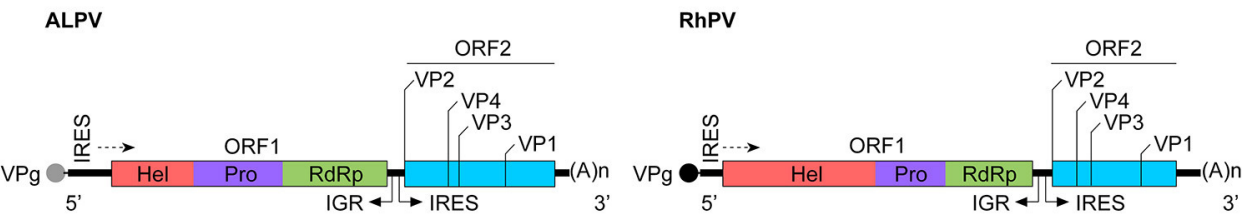

D

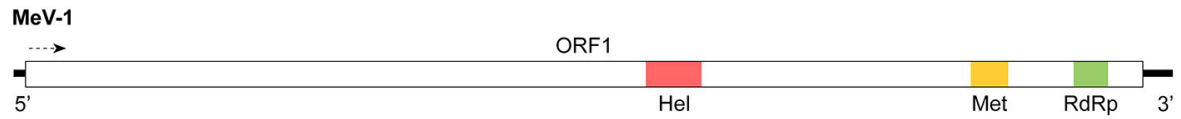

E

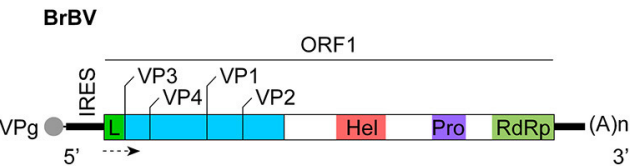

$\mathbf{F}$

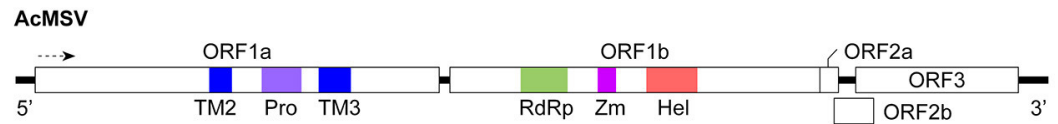

G

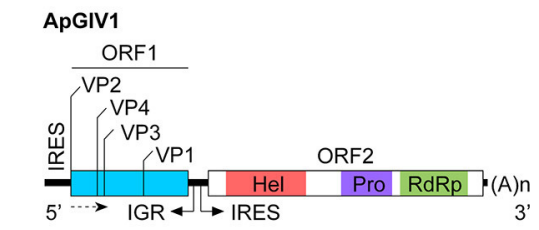

APV
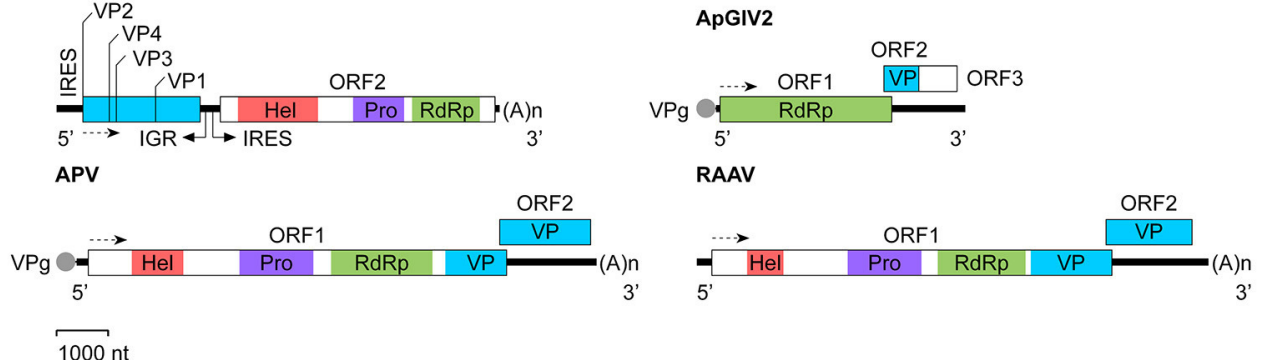

FIGURE 1 | Genomic organization and expression strategies of aphid viruses. The virus genomes used were: (A) Densoviruses: DpIDV (Dysaphis plantageinea densovirus), MpDV (Myzus persicae densovirus), and MpnDV (Myzus persicae nicotianae densovirus); (B) Bunyaviruses: ABV-1 (Aphid bunyavirus 1) and AcBV (Aphis 
FIGURE 1 | citricidus bunyavirus); (C) Dicistroviruses: ALPV (Aphid lethal paralysis virus) and RhPV (Rhopalosiphum padi virus); (D) Flavivirus: MeV-1 (Macrosiphum euphorbiae virus 1); (E) Iflavirus: BrBV (Brevicoryne brassicae virus); (F) Mesonivirus: AcMSV (Aphis citricidus meson-like virus); (G) Unclassified RNA viruses: ApGIV1 (Aphis glycines virus 1), ApGIV2 (Aphis glycines virus 2), APV (Acyrthosiphon pisum virus), and RAAV (Rosy apple aphid virus). The GenBank accession no. of each viral genome sequence (see Table 1). The determined or putative Vpg protein at the $5^{\prime}$-end of the viral RNA genome is indicated as a black or gray circle. The transcription direction for each virus is showed as the dashed line with arrow. (A)n, poly(A) sequence; G, glycoprotein; G1, glycoprotein 1; G2, glycoprotein 2; Hel, RNA helicase; IGR, intergenic region; iTR, incomplete inverted terminal repeat; IRES, internal ribosomal entry site; L, L-protein; Met, methyltransferase; NS, non-structural protein; Nup, nucleoprotein; Pro, protease; RdRp, RNA-dependent RNA polymerase; TM2, transmembrane protein 2; TM3, transmembrane protein 3 ; VP, structural protein; VPg, virus genome-linked protein; VPLA2, virus phospholipase 2; Zm, zinc cluster-binding domain.

Currently, Bunyavirales contains 12 families, 4 subfamilies, 54 genera and 477 species (41).

Recently, two aphid bunyaviruses, ABV-1 and AcBV were separately identified in the laboratory cultured aphids A. pisum or Aphis citricidus. The genome sequences of both viruses retrieved from the transcriptome data of the aphids were single-stranded, negative-sense RNA that composed of three segments: $\mathrm{L}$ (ABV1, 7,317 nt; AcBV, 7,037 nt), M (ABV-1, 6,626; AcBV, 3,462 nt), and S (ABV-1, 1,874 nt; AcBV, 1,163 nt) (Table 1). Each segment of the virus genome contains one ORF, which separately encodes an RdRp (ABV-1) or an RdRp and an L-protein (AcBV) (L segment), the envelope glycoprotein(s) (M segment), and the nucleoprotein (S segment) (Figure 1B). Phylogenetic analysis of the RdRp domain indicated that $\mathrm{ABV}-1$ and $\mathrm{AcBV}$ are grouped to the family Phasmaviridae or Phenuiviridae, respectively $(15,16)$.

\section{Host Range and Transmission of Aphid Bunyaviruses}

Prior studies indicated that ABV-1 could infect a bunch of aphids, such as Aphis aurantii, A. citricidus, A. gossypii, A. pisum, A. spiraecola, M. persicae, R. padi, Rhodobium porosum, and $S$. avenae. This virus may be transmitted vertically from infected aphids to nymphs or horizontally via plants infested by infected aphids (15).

\section{Dicistroviruses \\ Classification and Characters of the Genome of Aphid Dicistroviruses}

Dicistroviruses belong to the family Dicistroviridae (42). Singlestranded positive-sense RNA genome with the length of 9$11 \mathrm{~kb}$ of these viruses are packaged in non-enveloped virion particles (25-30 $\mathrm{nm}$ in diameter). The 5'-terminus of the genome is covalently linked to a virus-encoded small protein VPg (virus genome-linked protein) and the 3 '-terminus is polyadenylated. In general, dicistroviruses contain two ORFs that connected by a short intergenic region (IGR). The ORF1 located on the 5'-proximal of the genome encodes the NS proteins: an RNA helicase, a cysteine protease, and the RNA-dependent RNA polymerase (RdRp), whereas ORF2 located on the 3'-proximal of the genome encodes a large polyprotein which is processed to generate VP1-VP4 structural proteins. In addition to these features, dicistroviruses also contain an internal ribosome entry site (IRES) in front of each ORF that required for directing translation initiation in an AUG-independent manner $(43,44)$.

Dicistroviruses can infect various arthropods, including the insects in diptera, hemiptera, hymenoptera, and orthoptera (44). Currently, only two aphid discistroviruses ALPV and RhPV were characterized. Both of the viruses were identified in $R$. padi many years ago and grouped with Drosophila $C$ virus and cricket paralysis virus in the genus Cripavirus of Dicistroviridae (Table 1) $(11,18,45,46)$. Similar with the other member of Dicistroviridae, the positive-sense RNA genome of aphid dicistroviruses with the length of 9,812 nt (ALPV) or 10,011 nt (RhPV) are packaged in about $26 \mathrm{~nm}$ virion particles. The NS proteins encoded by ALPV or RhPV ORF1 contian an RNA helicase, a cysteine protease, and an RdRp domains, whereas the protein precursor encoded by ORF2 may be cleaved to form four structural proteins (VP1VP4) (Figure 1C) $(18,46)$. Recently, different isolates of ALPV has been identified in diverse insects including aphids (Aphis nerii, A.fabae, A. pisum, R. padi) $(17,47,48)$, bees (Apis mellifera, Apis cerana, and Verpa velutina) (49-52), and beetles (Diabrotica virgifera) (17), in bats (Hipposideros caffer) (53), and in plants (54). Genome sequences analysis indicated that ALPV isolates obtained from A. pisum and A. mellifera repectively are closely related, but both of them have relatively low sequence identities with those isolated from other insects and bats, suggesting that the isolates of ALPV from different arthropods may represent distinct ALPV strains or species (17).

\section{Infection Symptoms and Mechanisms of Aphid Dicistroviruses}

Under laboratory conditions, infection of ALPV and RhPV usually causes substantially distinct pathological symptoms in different aphids. For $R$. padi, the virus infection results in a short lifespan of the aphids, significant reduction of the fecundity, high mortality of nymphs, and a substantial decrease of population density $(55,56)$. In addition, ALPV infection also causes uncoordinated movement and paralysis of $R$. padi (45). Interestingly, RhPV infection was found to affect olfactory behavior of $R$. padi and cause infected aphids easily be predated or attacked by natural enemies (57). In contrast, an ALPV isolate identified from the wild population of the milkweed aphid $A$. nerii in northern Israel is asymptomatic in $A$. nerii, however, the virus could infect $M$. persicae and cause high mortality of the aphid (48). In an earlier field investigation, Laubscher and Von Wechmar found that a dramatic decline in grain aphids $R$. padi and $S$. avenae numbers were coincided with the high incidence of ALPV present in the aphid population, suggesting that ALPV could serve as a population growth-limiting factor in controlling aphids (56).

Current knowledge of infection mechanisms of aphid dicistroviruses is very limited. Earlier TEM analysis revealed that RhPV infection occurs in the posterior midgut cells or hindgut cells of infected aphids, and the infection was not observed in the anterior midgut (stomach), salivary gland, 
tracheal, nerve or muscle cells. Within the gut cells, RhPV replicates in the cytoplasm and the infection leads to the absence of the cytoplasmic organelles, except for mitochondria. Virions were found free or packed in crystalline arrays in large vesicles in the cytoplasm (58). Similar cytopathic effect (CPE) was also observed in RhPV-infected GWSS-Z10 cells (59), which are derived from the embryos of Homalodisca coagulate (60). Since the mitochondria and nuclear membranes remained intact during infection and the virus particles were not observed in the nuclei, these data imply that RhPV may replicate and assemble in the gut cells mitochondria.

\section{Host Range and Transmission Modes of Aphid Dicistroviruses}

Dicistroviruses have a variety of hosts (44). Earlier studies have demonstrated that RhPV could infect a narrow range of economically important grain aphids, including A. dirrhodum, Diuraphis noxia, R. maidis, $R$. padi, R. rufiabdominalis, and $S$. graminum (55). In contrast, the host range of ALPV is complicated. This virus was found to infect several distinct aphid species, such as A. nerii, M. dirhodum, M. persicae, R. padi, $S$. avenae, and $S$. graminum, and the whitefly $T$. vaporatiorum $(48,58,61)$. In addition, as mentioned above, deep-sequencing analysis indicated that ALPV or ALPV-like viruses were present in other insects including bees and beetles, in bats, and in plants $(17,54,62)$. Although the infection and pathogenecity of the virus in those animals and plants remain unclear, the high sequence similarity among different ALPV isolates suggest that ALPV-like viruses may have a wide range of hosts.

RhPV can actively spread among different individuals of aphids and plants play an important role in mediating the virus horizontal transmission. It was found that RhPV was present in all parts of the plant which were infested by infected aphids (63). Although RhPV seems to replicate less likely in plants, host plants may act as a virus reservoir and promote virus circulating in the aphid population $(63,64)$. Also, the virus may temporarily exist in plants during the winter season when aphids are absent in the environment. RhPV can be transmitted vertically from adult aphids to nymphs and the virus has been detected in different life stages of $R$. padi, including sexual aphids and eggs $(55,63)$. However, the transovarial passage rate of the virus was found to be substantially lower than that in horizontal transmission (55), which might be caused by the lower survival rate of nymphs produced by infected aphids.

\section{Flaviviruses}

\section{Classification and Characters of the Genome of Aphid Flaviviruses}

Flaviviruses are grouped in the genus flavivirus in the family Flaviviridae. The single-stranded positive-sense RNA genome, $9.2-11 \mathrm{~kb}$ in length, is assembled in small, enveloped virions (about $50 \mathrm{~nm}$ in diameter). The 5'-end of the genome is blocked with an m-7GpppAmp cap and the 3'-end is lack of a polyadenylated tail. Flaviviruses possess a long ORF that encodes a polyprotein, which is subsequently processed to generate structural and non-structural proteins. Structural proteins (VPs) are located at the $\mathrm{N}$-proximal proportion of the polyprotein, whereas NS proteins including an RNA helicase, a serine protease, and the RdRp are located at the C-terminal region (65).

Recently, a putative aphid flavivirus, named as Macrosiphum euphorbiae virus $1(\mathrm{MeV}-1)$, was identified in the potato aphid Macrosiphum euphorbiae. The genome of MeV-1 retrieved from the transcriptome data of $M$. euphorbiae is $22,780 \mathrm{nt}$ and contains a single large ORF encoding a polyprotein of 7,333 amino acids. Homology analysis indicated that the polyprotein of MeV-1 contains the helicase, methyltransferase, and RdRp domains (Figure 1D). Phylogenetic analysis of the helicase or $\mathrm{RdRp}$ domain of $\mathrm{MeV}-1$ indicated that this virus belongs to Flaviviridae. The presence of the methyltransferase domain in the polyprotein indicates that $\mathrm{MeV}-1$ is a putative member of the genus Flavirivus (Table 1) (19).

\section{Infection Symptoms and Transmission of Aphid Flaviviruses}

No obvious infection symptoms or abnormal phenotypes were observed in MeV-1 infected M. euphorbiae. Viral RNA and its replication intermediates were detected in all developmental stages of aphids and all newborn nymphs collected after laid off and grew on natural tomato plants, and the virus was not integrated into the M. euphorbiae genome, suggesting that $\mathrm{MeV}$ 1 can be transmitted vertically. In addition, $\mathrm{MeV}-1$ was detected in aphids-infested plant leaves, but the virus titer reduced dramatically after removal of the aphids, indicating that the virus could not replicate in plants and the presence of the virus in plants may contribute to the horizontal transmission of $\mathrm{MeV}$ 1 (19).

\section{Iflaviruses \\ Classification and Characters of the Genome of Aphid Iflaviruses}

Iflaviruses, a group of non-enveloped, small RNA viruses with the virion size ranging from 20 to $30 \mathrm{~nm}$ in diameter, belong to the family Iflaviridae. The single-stranded positive-sense RNA genome with the length of $8-10 \mathrm{~kb}$ contains a single ORF, which encodes a polyprotein that is subsequently processed to yield NS and VP proteins. NS proteins that generated by the C-terminal region of the polyprotein function as an RNA helicase, a 3C-like cysteine protease, and an RdRp. Four VP proteins (VP1-VP4) produced by the $\mathrm{N}$-terminal region of the polyprotein coordinate to form the virion capsid. Iflaviruses replicate in the cytoplasm of host cells, but the molecular mechanism of the virus infection remain unknown yet (66).

Currently, there is only one aphid iflavirus, Brevicoryne brassicae virus (BrBV), was identified from the infected cabbage aphid Brevicoryne brassicae (Table 1) (20). The single-stranded positive-sense RNA genome of $\mathrm{BrBV}, 10,161 \mathrm{nt}$ in length, contains a single ORF, which is predicted to encode a polyprotein of 2,983 amino acids. Sequence alignments indicated that the Nterminal region of $\mathrm{BrBV}$ polyprotein has high similarities with the VP proteins of other iflaviruses, whereas the C-terminal region of the polyprotein contains distinct regions of sequence identical to the RNA helicase, a chymotrypsin-like protease, and an RdRp (Figure 1E). Phylogenetic analysis revealed that $\mathrm{BrBV}$ is grouped together with Dinocampus coccinellae paralysis 
virus (20). Recently, a new isolate of BrBV (BrBV-IL) has been identified in aphids $B$. brassicae collected from wild mustard plants Sinapis arvensis in Israel. BrBV-IL genome shares a 95\% nucleotide sequence identity with that of BrBV (67).

\section{Infection Mechanisms of Aphid Iflaviruses}

To date, infection mechanisms of BrBV remain unclear, but the virus may not persist or replicate in Brassica and Arabidopsis plants. Intriguingly, the natural population of $B$. brassicae with a heavy infeciton of BrBV was less parasitized by parasitoid wasps, implying that $\mathrm{BrBV}$ infection affects the interaction between $B$. brassicae and its natural enemies (20).

\section{Mesoniviruses Classification and Characters of the Genome of Aphid Mesoniviruses}

Mesoniviruses, a group of enveloped, single-stranded positivesense RNA viruses that classified into the family Mesoniviridae, have a genome of $\sim 20 \mathrm{~kb}$ that contains 6-7 ORFs. Two large overlapped ORFs (ORF1a and ORF1b) located on the 5'-proximal of the genome $(\sim 15 \mathrm{~kb})$ encodes the NS proteins, which are arranged in order as the $3 \mathrm{C}$-like protease (3CLpro, encoded by ORF1a), RdRp, zinc-binding module and associated helicase (ZnHel1), exoribonuclease (ExoN), $\mathrm{N}^{7}$ methyltransferase (NMT), and 2'-O-methytransferase (OMT) (encoded by ORF1b). The other ORFs located at the 3'-terminus of the genome encode the structural proteins, including the spike (S) protein and other membrane proteins (68). Mesoniviruses have been isolated from infected mosquitoes and these viruses seems non-infectious to vertebrates $(68,69)$.

Recently, a new mesonivirus, AcMSV, has been identified in the brown citrus aphid $A$. citricidus (21). The viral genome retrieved from the transcriptome data of $A$. citricidus, 20,300 nt in length, contains five ORFs encoding six conserved proteins, including transmembrane protein 2 (TM2), a protease, TM3, an RdRp, a zinc cluster-binding domain (Zm), and an RNA helicase (Figure 1F). Phylogenetic analysis using the RdRp domain of AcMSV showed that this virus belongs to Mesoniviridae (Table 1) (21).

\section{Unclassified RNA Viruses Acyrthosiphon Pisum Virus \\ Classification and Characters of the Genome of APV}

APV is a positive-sense, single-stranded RNA virus with the genome of 10,016 nt (Table 1). The virion is about $31 \mathrm{~nm}$ in diameter and composed of four VP proteins with the molecular weight of 23.3, 24.2, 34.5, and $66.2 \mathrm{kDa}$. Sequence analysis indicated that APV genome contains two ORFs. The large ORF1 $(7,890 \mathrm{nt})$ is located on the 5'-proximal of the genome and predicted to encode a polyprotein of $296.3 \mathrm{kDa}$, which possesses the picorna-like RNA helicase, chymotrypsinlike cysteine protease, and RdRp domains. The small ORF2 (1,665 $\mathrm{nt}$ ) is located at the 3 '-end of the genome and overlaps the ORF1 by $28 \mathrm{nt}$. It's predicted to encode a protein of $63.3 \mathrm{kDa}$. Amino acid sequencing analysis showed that the capsid proteins of APV are produced by the 3'-half of the genome (Figure 1G). They may encode by the 3 '-end of ORF1 $(34.5 \mathrm{kDa})$ or ORF1 and ORF2
$(66.2 \mathrm{kDa})$, or generate by the proteolytic cleavage of the $34.5 \mathrm{kDa}$ protein (23.3 and $24.2 \mathrm{kDa}$ ). In addition, a subgenomic RNA of $\sim 4 \mathrm{~kb}$ was detected in both purified APV and the virus-infected aphids (22).

Infection Impact, Cytopathology, and Transmission of APV Although APV infection inhibited A. pisum nymphs growth and prolonged the development time (70), recent studies suggested that the virus infection may play a beneficial role in mediating adaption of aphids to low-fitness plants (71). During infection, APV was predominantly present in salivary glands and the gut epithelial cells of $A$. pisum nymphs $(70,71)$, but also observed in muscle cells and mycetocytes (70). It seems that the virus replicates in the cytoplasm and virions may be clustered in small vesicles. APV could not replicate in plants, but it can be persisted in plants for a few days and allowed horizontal transmission to aphids. The virus was found to be vertically transmitted to offspring of A. pisum. However, the transovarial transmission rate of APV was substantially low, which might be caused by the low level of the virus in ovaries of adult aphids (70).

\section{Aphis Glycines Virus 1}

ApGlV1 was identified in the field population of A. glycines. The viral genome is a single-stranded bicistronic RNA with the length of 8,680 nt (excluding the 3'-end polyadenylated tail) (Table 1). The genome contains two large ORFs, which are separated by an IGR fragment of $474 \mathrm{nt}$. In front of each ORF, there is an IRES element required for translation. ORF1 located on the 5'-proximal of the genome encodes four VP proteins, whereas the 3'-proximal ORF2 encodes a polyprotein containing an RNA helicase, a protease, and an RdRp domains (Figure 1G). The genomic structure of ApGlV1 is similar with that of dicipiviruses in the family Picornaviridae. Phylogenetic analysis indicated that ApGlV1 is grouped with the unclassified picorna-like viruses (23).

\section{Aphis Glycines Virus 2}

ApGlV2 was identified in the laboratory cultured A. glycines. ApGlV2 genome is a single-stranded positive-sense RNA with the length of about 4,850 nt (Table 1), which contains two predicted overlapping ORFs. ORF1 encodes a putative replicase of 1,121 amino acids, possessing the RdRp domain. ORF2 is predicted to encode a capsid protein (P24). In addition, at the downstream of the coding region of P24, there may exist an additional ORF3 which encodes a protein with the unknown function (Figure 1G). Although the RdRp domain of ApGlV2 showed low amino acid sequence similarities with the other RNA viruses, structure analysis suggested that the predicted tertiary structure of ApGIV2 RdRp was similar to those of tetraviruses and Drosophila A virus. ApGlV2 is not integrated into the genome of A. glycines. The virus could be transmitted vertically, but the horizontal transmission mode remain unknown yet (24).

\section{Rosy Apple Aphid Virus \\ Classification and Characters of the Genome of RAAV}

RAAV was detected in $D$. plantageinea. Virion particles of this virus are about $32 \mathrm{~nm}$ in diameter. The viral genome 
is a single-stranded positive-sense RNA with the length of 9,992 nt (Table 1). Similar with that of APV, RAAV contains two ORFs. The $5^{\prime}$-proximal large ORF1 (7,956 nt) is predicted to encode a polyprotein possessing the picorna-like RNA helicase, chymotrypsin-like cysteine protease, and RdRp domains. Additionally, the $3^{\prime}$-end of ORF1 may produce a small capsid protein. The $3^{\prime}$-proximal small ORF2 (1,692 nt) is overlapped the ORF1 by $54 \mathrm{nt}$ and predicted to encode another capsid protein (Figure 1G). RAAV has a high nucleotide sequence identity with APV.

\section{Transmission of RAAV}

The virus can be vertically transmitted to offspring of the aphid, but the transmission rate was found to be extremely low. In addition, virus-free aphids that placed on leaves far from the infected-aphids contaminated leaves became RAAV positive, suggesting that this virus could be horizontally transmitted via plants (12).

\section{CONCLUSION AND PERSPECTIVES}

In their complex life cycles, aphids, like other animals, are widely attacked by viruses. Compared with the other insect viruses, such as the extensively studied baculoviruses, the knowledge and research system of aphid pathogenic viruses are limited.

In recent years, next-generation sequencing and metagenomic analysis accelerate the finding of new aphid viruses (15, 21, 50, 62). Virus mining using viral transcripts or genomic sequences retrieved from the aphid genome or transcriptome data can save time spent on isolating or purifying virus particles, which usually need to collect a large scale of infected aphids. In addition, deepsequencing analysis is profoundly useful for identifying viruses that cause a chronic infection and/or atypical symptoms in aphids and evaluating integration of viral elements into aphid genomes.

Although a few aphid viruses, such as ALPV, MpDV, and $\mathrm{RhPV}$, showed dramatic negative impact on aphids growth or development, there is no application of these viruses as biopesticides for aphid control. A limitation is the obstacle to produce large quantities of virus. Using other insect viruses, such as the baculovirus expression system, or deleloping permissive

\section{REFERENCES}

1. Favret C, Eades DC. Aphid Species File (2021). Available online at: http:// Aphid.SpeciesFile.org (accessed January 20, 2022).

2. Loxdale HD, Balog A. Aphid specialism as an example of ecologicalevolutionary divergence. Biol Rev. (2018) 93:642-57. doi: 10.1111/brv.12361

3. Simon JC, Peccoud J. Rapid evolution of aphid pests in agricultural environments. Curr Opin Insect Sci. (2018) 26:1724. doi: 10.1016/j.cois.2017.12.009

4. Dedryver CA, Le Ralec A, Fabre F. The conflicting relationships between aphids and men: a review of aphid damage and control strategies. Comptes Rendus Biol. (2010) 333:539-53. doi: 10.1016/j.crvi.2010.03.009

5. Goggin FL. Plant-aphid interactions: molecular and ecological perspectives. Curr Opin Plant Biol. (2007) 10:399-408. doi: 10.1016/j.pbi.2007.06.004

6. Brault V, Uzest M, Monsion B, Jacquot E, Blanc S. Aphids as transport devices for plant viruses. Comptes Rendus Biol. (2010) 333:524-38. doi: 10.1016/j.crvi.2010.04.001 cell lines to produce aphid viruses will be useful for large-scale virus production. Alternatively, generating transgenic plants to express aphid viruses may promote host plants defensing against aphids. In addition, infection of RhPV was found to be beneficial to the predation or attack of natural enemies by affecting the olfactory behavior of aphids R. padi (57), this implies that the use of aphid viruses may enhance the control effect of natural enemies on aphids.

Currently, viral infection mechanisms and effects of the virus on field population dynamics of aphids are largely unknown. Intriguingly, prior studies on aphid densoviruses and APV suggested that the virus infection or the virus-derived sequences integrated in certain aphid genomes play important roles in regulating aphids development and dispersal, or promoting aphids adaption to low-fitness plants $(12,36,71)$. These pioneering studies will facilitate future investigation of the complex interaction of aphid viruses, aphids and the host plant. In addition, in vitro cultured cell lines of aphids are urgent to characterize the viral genome replication, gene function and virus particles assembly. It is worth noting that, although some aphid viruses, such as ALPV and RhPV, cause acute infection in certain aphids $(17,55,61)$, they have been found in other animals, including bees, bettles, and bats, or in plants $(17,50,53,54)$, raising a question about the host range and pathogenecity of these and other aphid viruses. Taken together, the systematic study of aphid viruses will not only provide new strategies for controlling aphids population, but also contribute to the protection of beneficial organisms including bees and other invertebrates.

\section{AUTHOR CONTRIBUTIONS}

YG and ZL drafted the manuscript. All authors contributed to revising the manuscript. All authors contributed to the article and approved the submitted version.

\section{FUNDING}

This work was supported by Chinese Universities Scientific Fund (Z1090121096, NWAFU) and the grant from National Key R\&D Program of China (2017YFC1200605).
7. Jayasinghe WH, Akhter MS, Nakahara K, Maruthi MN. Effect of aphid biology and morphology on plant virus transmission. Pest Manag Sci. (2021) 78:416-27. doi: 10.1002/ps.6629

8. Whitfield AE, Falk BW, Rotenberg D. Insect vector-mediated transmission of plant viruses. Virology. (2015) 479-80:278-89. doi: 10.1016/j.virol.2015.03.026

9. Peters D. The purification of viruslike particles from the aphid Myzus persicae. Virology. (1965) 26:159-61. doi: 10.1016/0042-6822(65) 90041-3

10. Parrish WB, Briggs JD. Morphological identification of viruslike particles in the corn leaf aphid, Rhopalosiphum maidis (Fitch). J Invertebrate Pathol. (1966) 8:122-3. doi: 10.1016/0022-2011(66)90111-X

11. D'Arcy CJ, Burnett PA, Hewings AD, Goodman RM. Purification and characterization of a virus from the aphid Rhopalosiphum padi. Virology. (1981) 112:346-9. doi: 10.1016/0042-6822(81)90 640-1

12. Ryabov EV, Keane G, Naish N, Evered C, Winstanley D. Densovirus induces winged morphs in asexual clones of the rosy apple 
aphid, Dysaphis plantaginea. Proc Natl Acad Sci USA. (2009) 106:8465-70. doi: 10.1073/pnas.0901389106

13. van Munster M, Dullemans AM, Verbeek M, van den Heuvel JFJM, Reinbold $\mathrm{C}$, Brault V, et al. A new virus infecting Myzus persicae has a genome organization similar to the species of the genus Densovirus. J Gen Virol. (2003) 84:165-72. doi: 10.1099/vir.0.18650-0

14. Tang S, Song X, Xue L, Wang X, Xu P, Ren G. Characterization and distribution analysis of a densovirus infecting Myzus persicae nicotianae (Hemiptera: Aphididae). J Econ Entomol. (2016) 109:580-77. doi: 10.1093/jee/tov399

15. An X, Zhang W, Ye C, Smagghe G, Wang JJ, Niu J. Discovery of a widespread presence bunyavirus that may have symbiont-like relationships with different species of aphids. Insect Sci. (2021) 1-6. doi: 10.1111/1744-7917.12989

16. Zhang W, Wu TF, Guo MM, Chang TY, Yang L, Tan Y, et al. Characterization of a new bunyavirus and its derived small RNAs in the brown citrus aphid, Aphis citricidus. Virus Genes. (2019) 55:55761. doi: 10.1007/s11262-019-01667-x

17. Liu S, Vijayendran D, Carrillo-Tripp J, Miller WA, Bonning BC. Analysis of new aphid lethal paralysis virus (ALPV) isolates suggests evolution of two ALPV species. J Gen Virol. (2014) 95:2809-19. doi: 10.1099/vir.0.0 69765-0

18. Moon JS, Domier LL, McCoppin NK, D'Arcy CJ, Jin N. Nucleotide sequence analysis shows that Rhopalosiphum padi virus is a member of a novel group of insect-infecting RNA viruses. Virology. (1998) 243:5465. doi: 10.1006/viro.1998.9043

19. Teixeira M, Sela N, Ng J, Casteel CL, Peng HC, Bekal S, et al. A novel virus from Macrosiphum euphorbiae with similarities to members of the family Flaviviridae. J Gen Virol. (2016) 97:1261-71. doi: 10.1099/jgv. 0.000414

20. Ryabov EV. A novel virus isolated from the aphid Brevicoryne brassicae with similarity to Hymenoptera picorna-like viruses. J Gen Virol. (2007) 88:2590-5. doi: 10.1099/vir.0.83050-0

21. Chang TY, Guo MM, Zhang W, Niu JZ, Wang JJ. First report of a mesonivirus and its derived small RNAs in an aphid species Aphis citricidus (Hemiptera: Aphididae), implying viral infection activity. J Insect Sci. (2020) 20:14. doi: 10.1093/jisesa/ieaa022

22. van der Wilk F, Dullemans AM, Verbeek M, van den Heuvel JF. Nucleotide sequence and genomic organization of Acyrthosiphon pisum virus. Virology. (1997) 238:353-362. doi: 10.1006/viro.1997.8835

23. Yasmin T, Thekke-Veetil T, Hobbs HA, Nelson BD, McCoppin NK, LagosKutz D, et al. Aphis glycines virus 1 , a new bicistronic virus with two functional internal ribosome entry sites, is related to a group of unclassified viruses in the Picornavirales. J Gen Virol. (2020) 101:10511. doi: 10.1099 /jgv. 0.001355

24. Liu S, Vijayendran D, Chen Y, Bonning BC. Aphis glycines virus 2, a novel insect virus with a unique genome structure. Viruses. (2016) 8:315. doi: 10.3390/v8110315

25. Tijssen P, Penzes JJ, Yu Q, Pham HT, Bergoin M. Diversity of small, singlestranded DNA viruses of invertebrates and their chaotic evolutionary past. $J$ Invertebr Pathol. (2017) 147:23-36. doi: 10.1016/j.jip.2017.06.008

26. Cotmore SF, Agbandje-Mckenna M, Chiorini JA, Mukha DV, Pintel DJ, Qiu JM, et al. The family Parvoviridae. Arch Virol. (2014) 159:123947. doi: 10.1007/s00705-013-1914-1

27. van Munster $M$, Dullemans AM, Verbeek $M$, van den Heuvel JFJM, Reinbold C, Brault V, et al. Characterization of a new densovirus infecting the green peach aphid Myzus persicae. $J$ Invertebrate Pathol. (2003) 84:6-14. doi: 10.1016/S0022-2011(03)00 013-2

28. Teixeira MA, Sela N, Atamian HS, Bao E, Chaudhary R, MacWilliams J, et al. Sequence analysis of the potato aphid Macrosiphum euphorbiae transcriptome identified two new viruses. PLoS ONE. (2018) 13:e0193239. doi: 10.1371/journal.pone.019 3239

29. Blair CD, Olson KE, Bonizzoni M. The widespread occurrence and potential biological roles of endogenous viral elements in insect genomes. Curr Issues Mol Biol. (2020) 34:13-30. doi: 10.21775/cimb.034.013

30. Frank JA, Feschotte C. Co-option of endogenous viral sequences for host cell function. Curr Opin Virol. (2017) 25:81-9. doi: 10.1016/j.coviro.2017.07.021
31. Johnson WE. Origins and evolutionary consequences of ancient endogenous retroviruses. Nat Rev Microbiol. (2019) 17:35570. doi: 10.1038/s41579-019-0189-2

32. Simmonds P, Aiewsakun P, Katzourakis A. Prisoners of war-host adaptation and its constraints on virus evolution. Nat Rev Microbiol. (2019) 17:3218. doi: 10.1038/s41579-018-0120-2

33. Wallau GL. RNA virus EVEs in insect genomes. Curr Opin Insect Sci. (2021) 49:42-7. doi: 10.1016/j.cois.2021.11.005

34. Brisson JA. Aphid wing dimorphisms: linking environmental and genetic control of trait variation. Philosophical Trans R Soc B Biol Sci. (2010) 365:60516. doi: $10.1098 /$ rstb.2009.0255

35. Liu H, Fu Y, Xie J, Cheng J, Ghabrial SA, Li G, et al. Widespread endogenization of densoviruses and parvoviruses in animal and human genomes. J Virol. (2011) 85:9863-76. doi: 10.1128/JVI.00828-11

36. Parker BJ, Brisson JA. A laterally transferred viral gene modifies aphid wing plasticity. Curr Biol. (2019) 29:2098-103. doi: 10.1016/j.cub.201 9.05.041

37. Clavijo G, van Munster M, Monsion B, Bochet N, Brault V. Transcription of densovirus endogenous sequences in the Myzus persicae genome. J Gen Virol. (2016) 97:1000-9. doi: 10.1099/jgv.0.000396

38. van Munster M, Janssen A, Clerivet A, van den Heuvel J. Can plants use an entomopathogenic virus as a defense against herbivores? Oecologia. (2005) 143:396-401. doi: 10.1007/s00442-004-1818-6

39. Wichgers Schreur PJ, Kormelink R, Kortekaas J. Genome packaging of the Bunyavirales. Curr Opin Virol. (2018) 33:1515. doi: 10.1016/j.coviro.2018.08.011

40. Maes P, Alkhovsky SV, Bao Y, Beer M, Birkhead M, Briese T, et al. Taxonomy of the family Arenaviridae and the order Bunyavirales: update 2018. Arch Virol. (2018) 163:2295-310. doi: 10.1007/s00705-018-3843-5

41. Abudurexiti A, Adkins S, Alioto D, Alkhovsky SV, Avsic-Zupanc T, Ballinger MJ, et al. Taxonomy of the order Bunyavirales: update 2019. Arch Virol. (2019) 164:1949-65. doi: 10.1007/s00705-019-04253-6

42. Valles SM, Chen Y, Firth AE, Guerin DMA, Hashimoto Y, Herrero S, et al. ICTV virus taxonomy profile: Dicistroviridae. J Gen Virol. (2017) 98:3556. doi: $10.1099 /$ jgv. 0.000756

43. Domier LL, McCoppin NK. In vivo activity of Rhopalosiphum padi virus internal ribosome entry sites. J Gen Virol. (2003) 84:415-9. doi: 10.1099/vir.0.18781-0

44. Bonning BC, Miller WA. Dicistroviruses. Annu Rev Entomol. (2010) 55:12950. doi: 10.1146/annurev-ento-112408-085457

45. Williamson C, Rybick EP, Kasdorf GGF, Von Wechmar MB. Characterization of a new picorna-like virus isolated from aphids. J Gen Virol. (1988) 69:78795. doi: 10.1099/0022-1317-69-4-787

46. van Munster M, Dullemans AM, Verbeek M, van den Heuvel JFJM, Clerivet A, van der Wilk F. Sequence analysis and genomic organization of Aphid lethal paralysis virus: a new member of the family Dicistroviridae. J Gen Virol. (2002) 83:3131-8. doi: 10.1099/0022-1317-83-12-3131

47. Wamonje FO, Michuki GN, Braidwood LA, Njuguna JN, Musembi Mutuku J, Djikeng A, et al. Viral metagenomics of aphids present in bean and maize plots on mixed-use farms in Kenya reveals the presence of three dicistroviruses including a novel Big Sioux River virus-like dicistrovirus. Virol J. (2017) 14:188. doi: 10.1186/s12985-017-0854-x

48. Dombrovsky A, Luria N. The Nerium oleander aphid Aphis nerii is tolerant to a local isolate of Aphid lethal paralysis virus (ALPV). Virus Genes. (2013) 46:354-61. doi: 10.1007/s11262-012-0846-2

49. Dalmon A, Gayral P, Decante D, Klopp C, Bigot D, Thomasson M, et al. Viruses in the invasive hornet Vespa velutina. Viruses. (2019) 11:1041. doi: 10.3390/v11111041

50. Granberg F, Vicente-Rubiano M, Rubio-Guerri C, Karisson OE, Kukielka D, Belak S, et al. Metagenomic detection of viral pathogens in Spanish honeybees: co-infection by Aphid lethal paralysis, israel acute paralysis and lake sinai viruses. PLoS ONE. (2013) 8:e57459. doi: 10.1371/journal.pone.0057459

51. Roberts JMK, Anderson DL, Durr PA. Metagenomic analysis of Varroa-free Australian honey bees (Apis mellifera) shows a diverse Picornavirales virome. J Gen Virol. (2018) 99:818-826. doi: 10.1099/jgv.0.001073

52. Yang D, Zhao H, Shi J, Xu X, Wu Y, Guo R, et al. Discovery of aphid lethal paralysis virus in Vespa velutina and Apis cerana in China. Insects. (2019) 10:157. doi: 10.3390/insects 10060157 
53. Bourgarel M, Noel V, Pfukenyl D, Michaux J, Andre A, Becquart P, et al. Next-generation sequencing on insectivorous bat guano: an accurate tool to identify arthropod viruses of potential agricultural concern. Viruses. (2019) 11:1102. doi: $10.3390 / \mathrm{v} 11121102$

54. Maina S, Edwards OR, de Almeida L, Ximenes A, Jones RA. Metagenomic analysis of cucumber RNA from East Timor reveals an aphid lethal paralysis virus genome. Genome Announcements. (2017) 5:e01445-16. doi: 10.1128/genomeA.01445-16

55. D'Arcy CJ, Burnett PA, Hewings AD. Detection, biological effects, and transmission of a virus of the aphid Rhopalosiphum padi. Virology. (1981) 114:268-72. doi: 10.1016/0042-6822(81)90275-0

56. Laubscher JM, Von Wechmar MB. Assessment of aphid lethal paralysis virus as an apparent population growth-limiting factor in grain aphids in the presence of other natural enemies. Biocontrol Sci Technol. (1993) 3:45566. doi: 10.1080/09583159309355300

57. Ban LP, Ahmed E, Ninkovic V, Delp G, Glinwood R. Infection with an insect virus affects olfactory behaviour and interactions with host plant and natural enemies in an aphid. Entomologia Experimentalis Et Applicata. (2008) 127:108-17. doi: 10.1111/j.1570-7458.2008.00675.x

58. Gildow FE, D'Arcy CJ. Cytopathology and experimental host range of Rhopalosiphum padi virus, a small isometric RNA virus infecting cereal grain aphids. J Invertebrate Pathol. (1990) 55:245-57. doi: 10.1016/0022-2011(90)90060-J

59. Boyapalle S, Pal N, Miller WA, Bonning BC. A glassy-winged sharpshooter cell line supports replication of Rhopalosiphum padi virus (Dicistroviridae). J Invertebrate Pathol. (2007) 94:130-9. doi: 10.1016/j.jip.200 6.09.010

60. Kamita SG, Do ZN, Samra AI, Hagler JR, Hammock BD. Characterization of cell lines developed from the glassy-winged sharpshooter, Homalodisca coagulata (hemiptera: cicadellidae). In vitro cellular \& developmental biology. Animal. (2005) 41:149-53. doi: 10.1290/0501002.1

61. Williamson C, Rybick EP, Von Wechmar MB. Further characterization of Rhopalosiphum padi virus of aphids and comparison of isolates from South Africa and Illinois. J Invertebrate Pathol. (1989) 54:8596. doi: 10.1016/0022-2011(89)90144-4

62. Zana B, Kemenesi G, Urban P, Foldes F, Jak ab F. Metagenomic analysis of bat guano samples revealed the presence of viruses potentially carried by insects, among others by Apis mellifera in Hungary. Acta Vet Hungarica. (2018) 66:151-61. doi: 10.1556/004.2018.014

63. Ban L, Didon A, Jonsson LM, Glinwood R, Delp G. An improved detection method for the Rhopalosiphum padi virus (RhPV) allows monitoring of its presence in aphids and movement within plants. J Virol Methods. (2007) 142:136-42. doi: 10.1016/j.jviromet.2007.01.014

64. Gildow FE, D'Arcy CJ. Barley and oats as reservoirs for an aphid virus and the influence on barley yellow dwarf virus transmission. Phytopathology. (1988) 78:811-6. doi: 10.1094/Phyto-78-811
65. Simmonds P, Becher P, Bukh J, Gould EA, Meyers G, Monath T, et al. ICTV virus taxonomy profile: Flaviviridae. J Gen Virol. (2017) 98:23. doi: 10.1099/jgv.0.000672

66. Valles SM, Chen Y, Firth AE, Guerin DMA, Hashimoto Y, Herrero S, et al. ICTV virus taxonomy profile: Iflaviridae. J Gen Virol. (2017) 98:5278. doi: 10.1099/jgv.0.000757

67. Luria N, Reingold V, Lachman O, Sela N, Dombrovsky A. Extended phylogenetic analysis of a new Israeli isolate of Brevicoryne brassicae virus (BrBV-IL) suggests taxonomic revision of the genus Iflavirus. Virol J. (2016) 13:50. doi: 10.1186/s12985-016-0500-Z

68. Lauber C, Ziebuhr J, Junglen S, Drosten C, Zirkel F, Nga PT, et al. Mesoniviridae: a proposed new family in the order Nidovirales formed by a single species of mosquito-borne viruses. Arch Virol. (2012) 157:1623-8. doi: 10.1007/s00705-012-1 295-x

69. Vasilakis N, Guzman H, Firth C, Forrester NL, Widen SG, Wood TG, et al. Mesoniviruses are mosquito-specific viruses with extensive geographic distribution and host range. Virol J. (2014) 11:97. doi: 10.1186/1743-422X-1 $1-97$

70. van den Heuvel JF, Hummelen H, Verbeek M, Dullemans AM, van der Wilk F. Characteristics of Acyrthosiphon pisum virus, a newly identified virus infecting the pea aphid. J Invertebrate Pathol. (1997) 70:16976. doi: 10.1006/jipa.1997.4691

71. Lu H, Zhu J, Yu J, Chen X, Kang L, Cui F. A symbiotic virus facilitates aphid adaptation to host plants by suppressing jasmonic acid responses. Molecular Plant Microbe Interact. (2020) 33:55-65. doi: 10.1094/MPMI-01-19-0 016-R

Conflict of Interest: The authors declare that the research was conducted in the absence of any commercial or financial relationships that could be construed as a potential conflict of interest.

Publisher's Note: All claims expressed in this article are solely those of the authors and do not necessarily represent those of their affiliated organizations, or those of the publisher, the editors and the reviewers. Any product that may be evaluated in this article, or claim that may be made by its manufacturer, is not guaranteed or endorsed by the publisher.

Copyright (C) $2022 \mathrm{Guo}, \mathrm{Ji}, \mathrm{Bai}, \mathrm{Ma}$ and Li. This is an open-access article distributed under the terms of the Creative Commons Attribution License (CC BY). The use, distribution or reproduction in other forums is permitted, provided the original author(s) and the copyright owner(s) are credited and that the original publication in this journal is cited, in accordance with accepted academic practice. No use, distribution or reproduction is permitted which does not comply with these terms. 